\title{
Metodología del estudio piloto
}

\author{
Gustavo Díaz-Muñoz ${ }^{*}$
}

1. Profesor. Programa de tecnología en radiología e imágenes diagnósticas. Fundación Tecnológica Autónoma de Bogotá. Bogotá, Colombia.

\section{Methodology of the pilot studies}

\section{Resumen:}

La enseñanza de la investigación debe abarcar todos los aspectos de su proceso, involucrando los estudios piloto. En la investigación biomédica, el primer paso en la ejecución de un proyecto es la realización de un estudio o prueba piloto, que busca probar en menor escala aspectos logísticos de la ejecución del estudio, lo que evitará cometer errores en los estudios posteriores y de mayor envergadura. La presente revisión pretende exponer aspectos fundamentales en la utilización y planeación de los estudios piloto, lo que servirá para optimizar los procesos de investigación en las áreas de la salud.

Palabras clave: Bioestadística; Educación médica; Metodología; Proyectos de investigación; Proyectos piloto; Revisión.

\section{Abstract:}

The teaching of research covers all aspects of the research process, involving pilot studies. In biomedical research, the first step in the execution of a project is the realization of a pilot study, with the objective of testing on smaller scale logistic aspects of the execution of the study, which will avoid making mistakes in the larger studies. This review aims to expose fundamental aspects in the use and planning of pilot studies, which will serve to optimize research processes in the areas of health.

Keywords: Bioestatistics; Medical Education; Methodology; Pilot Projects; Research design; Review.

Díaz-Muñoz G. et al. Metodología del estudio piloto. Rev Chil Radiol 2020; 26(3): 100-104.

*Correo electrónico: Gustavo Díaz-Muñoz / gustavo.diaz@correo.faba.edu.co

Trabajo enviado el 28 de marzo de 2020. Aceptado para publicación el 21 de junio de 2020.

\section{Introducción}

El uso y respectiva publicación de estudios piloto se ha incrementado constantemente en los últimos 20 años. En una búsqueda en pubmed.gov y empleando el término "Pilot study", se encontró que en el año 2000 existían 290 publicaciones, para el año 2010 existían 6.632 y en el 2019 se alcanzaron 11.716 publicaciones (Figura 1). Lo anterior describe una tendencia a hacia la mayor elaboración y publicación de estudios piloto. En el mismo sentido, este tipo de investigación es publicable en diversos tipos de revistas, generando que en el 2015 apareciera la primera revista especializada en este diseño de investigación: "Pilot and feasibility studies", ISSN:2055-5784 (Electronic)'.

Sin embargo, al parecer los estudios piloto reciben poca o nula importancia en el campo de la enseñanza de la investigación, encontrando que son pocos los textos que hacen mención y detalle al tema. Solamente algunos artículos (principalmente editoriales) y desde algunos campos de la salud se manifiestan aspectos metodológicos que deberían ser conocidos por todos los investigadores en ciencias de la salud ${ }^{2,3}$. 


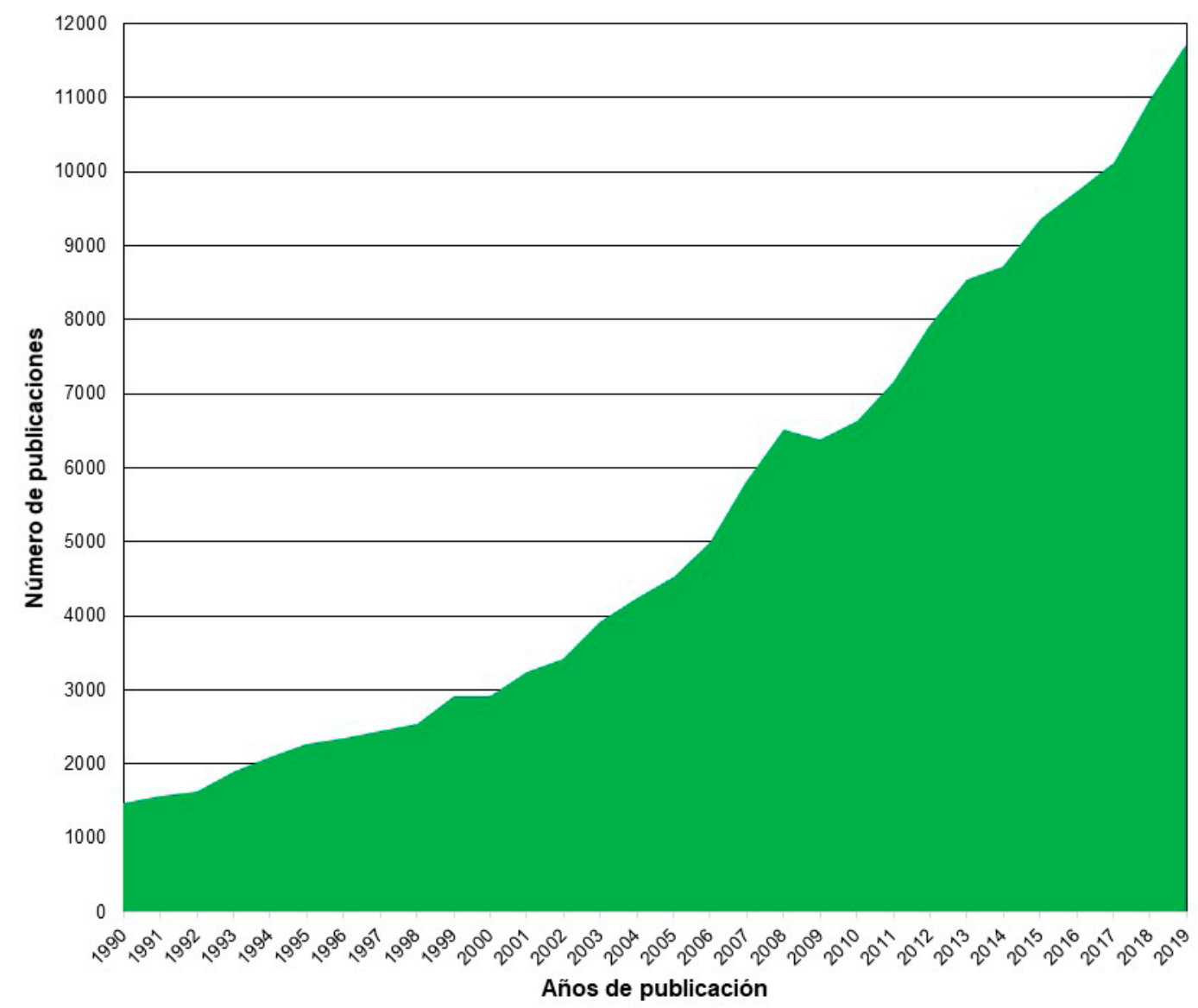

Figura 1: Número de estudios piloto publicados entre los años 1990 y 2019. Fuente: https://www.ncbi.nlm.nih.gov/

Junto a lo anterior, existen prácticas inadecuadas en la realización de los estudios piloto ${ }^{2,4}$, lo que genera reportes de artículos inapropiados y malas prácticas en el diseño de este tipo de investigación. Si se esclarecen los aspectos metodológicos de la realización de los estudios piloto, se podrán aprovechar al máximo las ventajas que trae la realización de este tipo de investigación, por lo que el objetivo de esta revisión es describir los aspectos relevantes para la realización de estudios piloto en el área de la salud.

\section{Metodología}

Para este artículo se realizó una revisión de la literatura en PubMed, empleando el algoritmo de búsqueda (Pilot study AND (methods or research design)) y se seleccionaron los artículos en primera instancia según el título y abstract a partir del año 2000. Se incluyeron artículos relacionados a aspectos del diseño y conducción de estudios piloto. El análisis de la información fue narrativo, enfocándose en los diversos aspectos del diseño de estudios y resumiéndolos en las secciones que se presentan a continuación.

\section{Definición}

La definición de estudio piloto no es unánime ${ }^{1}$ y se podría decir que varía conforme las necesidades de cada campo de investigación y del diseño de estudio, sin embargo, la mayoría de las definiciones coinciden en que permiten planear un estudio de mayor escala o magnitud ${ }^{2}$.

Un estudio piloto es un estudio pequeño o corto de factibilidad o viabilidad, conducido para probar aspectos metodológicos de un estudio de mayor escala, envergadura o complejidad ${ }^{2,5,6}$. La naturaleza de estos estudios es evitar la aparición de un defecto que sería nefasto en un estudio posterior que es costoso en recursos ${ }^{2,7}$. La definición permite inferir que no deberían diseñarse para responder preguntas o hipótesis de investigación, sino para responder preguntas de métodos específicos, es decir, evaluar la adecuación de los métodos y procesos, lo que evitará iniciar investigaciones de mayor escala sin un conocimiento o certeza del funcionamiento de los métodos que se proponen ${ }^{1,4,5,8}$.

Por lo anterior, se espera que un estudio piloto se desarrolle por razones de proceso (pasos que deben llevarse a cabo como parte del estudio principal), de recursos (tiempo y presupuesto necesarios para el estudio principal), de gestión (de recursos y datos) y científicas (mediciones y seguridad del participante) ${ }^{2}$. 
Al mismo tiempo, se pueden encontrar dos tipos de estudios piloto: 1.) el de viabilidad o factibilidad, que se enfoca en precisar si es realizable o posible el estudio principal; 2.) los piloto aleatorizados y no aleatorizados, que se asemejan al grupo anterior, pero serán el sustento para estudios de intervención más complejos. Sin embargo, también es imperativo diferenciarlos de los estudios exploratorios, éste último se conduce para comprobar en una menor escala una hipótesis de investigación, usualmente relacionada al conocimiento de los mecanismos o eficacia de alguna intervención ${ }^{9}$.

Considerando la definición de un estudio piloto, se pueden definir ${ }^{7}$ objetivos principales de un estudio piloto 1,10 :

1. Probar la integridad del protocolo de estudio para el futuro estudio

2. Obtener estimaciones iniciales para el cálculo del tamaño de la muestra

3. Probar formularios o cuestionarios de recolección de datos

4. Probar los procedimientos de asignación al azar

5. Estimar las tasas de reclutamiento y consentimiento

6. Determinar la aceptabilidad de la intervención

7. Seleccionar las medidas más apropiadas del desenlace primario.

Es factible que el estudio abarque uno o más objetivos, los cuales se pueden agrupar en dos tipos: objetivos logísticos y objetivos de viabilidad. Esta propuesta de clasificación permitirá orientar el alcance y planeación del estudio piloto, como se detalla más adelante.

\section{Objetivos logísticos}

Los objetivos logísticos de un estudio piloto se refieren a aspectos operativos y organizacionales de la investigación, abarcando los asuntos de evaluación de tiempos, necesidad de recursos y manejo de información ${ }^{8}$. La evaluación de tiempo se centra en contabilizar la duración de los procesos de la investigación: procedimiento de consentimiento informado, desplazamientos, tiempo en la búsqueda de participantes, sistematización de la información, entre otros.

La identificación de necesidades de recursos se refiere a: cantidad de personal, definir perfiles o funciones de cada ayudante de investigación, métodos de transporte (investigadores, muestras y/o participantes), permisos de acceso, entre otros. Respecto al manejo de información, se debe contemplar la pertinencia de los equipos de recolección ${ }^{8}$, disponibilidad de la información (registros, entrevistas, bases de datos, etc.), interpretación de preguntas/cuestionarios, aplicabilidad de los criterios de selección y cantidad de información completa.

\section{Objetivos de viabilidad}

Son los enfocados hacia responder preguntas relacionadas a la posibilidad de hacerse o no el estudio principal, como sería: porcentajes (reclutamiento, respuesta, retención y/o seguimiento), seguridad del paciente (en las mediciones y/o intervenciones), viabilidad de realizar una medición, posibilidad de acceder a la información u obtener un parámetro ${ }^{8}$.

Además, el estudio piloto ayuda a asegurar al financiador que el estudio se puede hacer y que es un estudio en que vale la pena invertir; la ejecución y finalización de un piloto permite observar la capacidad de trabajo del equipo de investigación, es decir, muestra que el equipo de investigación puede y ha trabajado en conjunto para completar con éxito el estudio piloto, lo cual es un buen indicio para el proyecto principal ${ }^{8}$.

Por otra parte, el estudio piloto también permite evaluar el lugar o sitio de ejecución del estudio ${ }^{8}$, lo cual incluye: la infraestructura, acceso a los participantes, disponibilidad de información y colaboración de los trabajadores del lugar.

\section{Planeación del estudio piloto}

La planeación del estudio piloto deberá ser tan rigurosa como en otros estudios ${ }^{1}$, debido a que aportará información valiosa para mejorar la metodología de investigaciones posteriores de mayor envergadura, es decir, no valdría la pena desarrollar un proyecto de investigación costoso a partir de información inválida o poco confiable. Además, la publicación de un estudio piloto es viable ${ }^{8}$, por lo que su planeación y detalle deberán estar al nivel de una publicación científica; de ahí que se consideren aspectos similares a los de un protocolo: problema, pregunta(s) de investigación, justificación, objetivos, materiales y métodos y resultados esperados.

También es relevante considerar su vínculo o nexo con el estudio posterior y de mayor envergadura, en otras palabras, es necesario mantener en mente el siguiente estudio o estudio principal. Por tanto, y considerando los objetivos del piloto, el protocolo del estudio piloto podría estar inmerso en el protocolo de investigación del estudio principal (usualmente en la sección de métodos o con un apartado en el anexo) o podría demandar un protocolo aparte y previo al estudio principal, que deberá ser revisado y aprobado por un comité de ética (según las normas de cada institución/país y el riesgo al que estén expuestos los participantes) ${ }^{11}$.

A sí mismo, si uno de los objetivos del estudio piloto es evaluar asuntos relacionados a las mediciones de la investigación principal, se deberán realizar mediciones de manera idéntica a las del estudio principal. Cabe destacar que, si una investigación involucra la aleatorización y/o ocultamiento, su ejecución en el estudio piloto deberá ser meditada, debido a que el 
mismo procedimiento podría o no ocultar en el piloto aspectos relevantes para la ejecución del estudio principal, como eventos adversos, pérdida de pacientes, adherencia a la intervención, etc.

\section{Tamaño de muestra}

Un aspecto fundamental en el estudio piloto es la cantidad mínima de sujetos a incluir, que sean suficientes para responder los objetivos del piloto y que representen un segmento pequeño de la investigación principal. Por lo tanto, en el cálculo del tamaño de muestra de los estudios piloto se deben considerar uno o ambos de los siguientes escenarios: a.) si los objetivos del piloto se relacionan a objetivos de viabilidad o logísticos, de manera subjetiva se debe estimar a priori la probabilidad de los eventos indeseables en el estudio; b.) si en el estudio piloto se desea obtener información para el cálculo de la muestra del estudio principal, para la estimación del número de sujetos se podrían modificar los valores relacionados al error estadístico (disminuir la confianza y el poder); estos dos escenarios se detallan en los siguientes párrafos.

El cálculo de tamaño de muestra empleado para identificar un problema o evento indeseable en el estudio piloto, se basa en una probabilidad a priori que el investigador dé a los eventos indeseados esperados; en este sentido, Viechtbauer et al. tomaron la probabilidad del evento a evaluar en el piloto y desarrollaron una fórmula para el tamaño de muestra en estudios piloto, la cual está disponible para su uso en la siguiente web: http://www.pilotsamplesize.com; para usar la fórmula, el investigador debe a priori determinar la confianza (valor de 0 a 1, idealmente superior a 0.95) y la probabilidad (valor entre 0 a 1) del evento a evaluar ${ }^{12}$.

Para el caso particular de obtener un parámetro a partir de un piloto que permita calcular el tamaño de muestra del estudio principal, se ha sugerido emplear las mismas fórmulas que se utilizarían en el proyecto de investigación de mayor envergadura, pero aumentando los valores de error a un nivel admisible, es decir, aumentar el valor de la significancia estadística (fijar valores por encima de 0.05) y disminuir el valor del poder (contemplar valores inferiores a 0.8). Por lo anterior, los resultados del estudio piloto deberán ser interpretados a la luz de dichas modificaciones y deberán asumirse que poseen cierto nivel error ${ }^{2,7,10}$.

\section{Recolección y análisis de la información}

En la recolección de información se debe reunir de manera paralela la información del estudio principal y datos sobre la ejecución del estudio, estos últimos serán relacionados al objetivo del estudio piloto y al cálculo del tamaño de muestra del piloto.

Además, el análisis de información usualmente no trasciende de un análisis descriptivo de la información (frecuencias, porcentajes, promedios, medianas y/o rangos). Es de recordar que la comparación de valores, prueba o contraste de hipótesis no es aconsejable en los estudio piloto, debido a que el tamaño de muestra y la rigurosidad del piloto (por ejemplo selección de participantes a conveniencia o la ausencia de enmascaramiento, ocultamiento y/o aleatorización) podrían afectar la validez de los resultados ${ }^{1,3,8}$. En el mismo sentido, se debe resaltar que la discusión de los resultados de un estudio piloto deberán relacionarse a la identificación de problemas en la ejecución del estudio y formular sus respectivas soluciones ${ }^{1,10}$.

Otro aspecto a considerar en el análisis es la inclusión de los participantes del estudio piloto en la investigación principal. Cada caso es diferente, debido a que se debe considerar la posibilidad que los participantes del piloto contaminen o alteren el principal; si hay modificación del protocolo del estudio posterior al piloto, la inclusión de los participantes sería inadecuado. También se debe contemplar que los participantes del piloto pueden representar parcialmente a los del estudio principal, debido a que ya sufrieron la exposición y/o aleatorización, lo que podría sesgar los resultados del estudio principal ${ }^{1,8,10}$.

\section{Publicación de un estudio piloto}

Los estudios piloto encajan perfectamente en la premisa de que no existen los estudios malos, ni estudios que no deban difundirse y todo estudio debería publicarse para que otros aprendan ${ }^{5,8}$. Hasta la fecha no existe una guía para el reporte de estudios piloto, sólo se dispone de la extensión CONSORT para estudio piloto de experimento o ensayos ${ }^{13}$.

Debido a lo anterior, al momento de publicar un estudio piloto se recomienda ${ }^{5,8,11,14}$ :

1. En el título y en el artículo declarar que es un estudio piloto.

2. En la introducción justificar la necesidad del estudio piloto.

3. Tener en mente que el estudio se centra en la viabilidad de realizar el estudio, no de realizar pruebas de hipótesis.

4. Incluir una sección de métodos: diseño de estudio, muestra, muestreo, reclutamiento, mediciones, intervenciones, análisis de datos y componente ético.

5. Los resultados deberán enfocarse en el objetivo del estudio piloto, por lo que el análisis estadístico tendrá un alcance descriptivo.

6. La discusión no debe sobre interpretar o inflar los resultados. Se debe centrar en la viabilidad del estudio, mediante la identificación de los problemas en la realización del estudio y cómo superarlos en la ejecución del estudio posterior o principal.

7. Resaltar las lecciones aprendidas y su implementación en estudios posteriores. 
8. Identificar las limitaciones del estudio y sesgos, con el fin de mantener al lector enfocado en que el estudio piloto evalúa la viabilidad de un estudio posterior y no está probando hipótesis de investigación.

\section{Lo que no es un estudio piloto}

Por último, en el artículo se han señalado aspectos del diseño del estudio piloto, pero a manera de reflexión, es importante resaltar lo que no es o no debería ser llamado estudio piloto $3,4,14,15$, por ejemplo los proyectos de investigación que por poca o nula financiación no alcanzaron a reclutar un número suficiente de participantes, investigaciones con un tamaño de muestra pequeño y/o con un rigor metodológico inadecuado, estudios que prueban o contrastan hipótesis a partir de muestras pequeñas y finalmente publicaciones sin objetivos o alcances que permitan avanzar en la ciencia.

\section{Conclusiones}

El estudio piloto es un paso esencial en el proceso de investigación, el cual se centra en evaluar los aspectos metodológicos y procedimentales de una investigación posterior de mayor escala, por tanto, su planeación, ejecución y divulgación deberá ser rigurosa. La publicación de los estudios piloto es pertinente, debido al alcance pedagógico que tienen para señalar y superar errores en el desarrollo de una investigación. Por lo anterior, los elementos de planeación, desarrollo y publicación de un estudio piloto deben estar a la altura de cualquier otro diseño de estudio.

Conflictos de interés: No hay conflictos de interés que declarar

Financiación: Fundación tecnológica autónoma de Bogotá. Bogotá, Colombia.

Colaboración individual: El autor participó en la creación de la idea de investigación, búsqueda y revisión de documentos, redacción del manuscrito y sometimiento del artículo.

\section{Referencias}

1. Lancaster GA. Pilot and feasibility studies come of age. Pilot Feasibility Stud. 2015; 1(1): 1-4.

2. Thabane L, Ma J, Chu R, Cheng J, Ismaila A, Rios LP, et al. A tutorial on pilot studies: The what, why and how. BMC Med Res Methodol. 2010; 10(1): 1-10.

3. Dworschak M, Campbell MJ. About the benefits and limitations of pilot studies. Minerva Anestesiol. 2015; 81(7): 711.

4. Lowe NK. What is a pilot study? J Obstet Gynecol Neonatal Nurs. 2019; 48(2): 117-118.

5. Doody O, Doody CM. Conducting a pilot study: Case study of a novice researcher. Br J Nurs. 2015; 24(21): 1074-1078.

6. Porta M. A dictionary of epidemiology. 5th ed. New York: Oxford University press; 2008: 316.

7. Kraemer HC, Mintz J, Noda A, Tinklenberg J, Yesavage JA. Caution regarding the use of pilot studies to guide power calculations for study proposals. Arch Gen Psychiatry. 2006; 63(5): 484-489.

8. Resnick B. The definition, purpose and value of pilot research. Geriatric Nursing. 2015; 36(2): S1-S2.

9. Rosenbloom DA, Morris NS. Defining and understanding pilot and other feasibility studies. Am J Nurs. 2017; 117(3): 38-45.

10. Lancaster GA, Dodd S, Williamson PR. Design and analysis of pilot studies: Recommendations for good practice. J Eval Clin Pract. 2004; 10(2): 307-312.

11. Moore CG, Carter RE, Nietert PJ, Stewart PW. Recommendations for planning pilot studies in clinical and translational research. Clin Transl Sci. 2011; 4(5): 332-337.

12. Viechtbauer W, Smits L, Kotz D, Budé L, Spigt M, Serroyen J, et al. A simple formula for the calculation of sample size in pilot studies. J Clin Epidemiol. 2015; 68(11): 1375-1379.

13. Abbade LPF, Abbade JF, Thabane L. Introducing the CONSORT extension to pilot trials: Enhancing the design, conduct and reporting of pilot or feasibility trials. J Venom Anim Toxins Incl Trop Dis. 2018; 24(1): 3 .

14. Foster RL. What a pilot study is and what it is not. J Spec Pediatr Nurs. 2013; 18(1): 1-2.

15. Leon AC, Davis LL, Kraemer HC. The role and interpretation of pilot studies in clinical research. J Psychiatr Res. 2010; 45(5): 626-629. 